Results The association between marital status and waist circumference was altered by living arrangement, social network size and social participation, with different combined effects for women and men. All non-partnered women who were co-living had higher mean WC than partnered women who were co-living. Mean WC was higher in lone-living than in co-living women in all marital status categories except for widowed. Lone-living widowed women had a lower WC $(-0.97 \mathrm{~cm}[-3.85,1.91])$, compared to lone-living partnered, that was significantly different (p-interaction $=0.005)$ from co-living widowed women $(+3.57[2.26$, $4.88])$ relative to co-living partnered women. We found that men who were partnered, single, or widowed had higher mean WC when they were also lone-living compared to counterparts who were co-living. Social network size was positively associated with mean WC in women for all marital status categories, except divorced, whereas social participation was inversely associated with mean WC in women for all marital status categories, especially divorced. Combined effects were less clear in men. Greater social participation (5 or more activities) also appeared to mitigate the health-harming influence of having a low social network size on WC in women but not men. Specifically, in the absence of social participation, mean WC reduced by 0.63 $\mathrm{cm}(-0.88,-0.38)$ in women and by $0.27 \mathrm{~cm}(-0.51,-0.03)$ in men as social network size increased. Notably, living arrangement did not appear to modify the link between either social participation or social network size and WC in either gender.

Conclusion The interplay of different types of social ties revealed an important source of heterogeneity with unique associations with visceral adiposity in women and men. Prevention efforts can be improved by understanding which modifiable social factors are most relevant for obesity in women and men.

\section{P59 THE EFFECT OF CHILDHOOD OBESITY AND OVERWEIGHT ON EDUCATIONAL OUTCOMES: AN INTERDISCIPLINARY SECONDARY ANALYSIS OF THREE UK COHORTS}

AB Segal* ${ }^{*}$ MC Huerta, F Sassi. Centre for Health Economics and Policy Innovation, Imperial College Business School, London, UK

\subsection{6/jech-2020-SSMabstracts. 152}

Background Childhood obesity has been shown to affect human capital and social outcomes later in life. Yet, evidence of the causal nature of this link is scarce and pathways are not well understood. We aimed to investigate the effect of childhood obesity on cognitive performance in adolescence and educational attainment in early adulthood.

Methods We used data of three longitudinal UK cohorts: the1958 National Child Development Study (NCDS; $\mathrm{n}=5346$ ), the 1970 British Cohort Study (BCS70; $\mathrm{n}=6790$ ) and the Avon Longitudinal Study of Parents and Children (ALSPAC; $\mathrm{n}=5373$ ) which includes children born in1991-92. We used ordinary least squares and logistic regression, valueadded sex-stratified models, and mendelian randomisation models to explore the effect of childhood body-mass index (BMI; Z score and BMI category at age 11 years or at 16 years) on cognitive performance (Maths and English scores at age 16 years) and educational attainment (tertiary qualification at age 23 years).
Results In ALSPAC, female individuals who had been overweight at age 11 years scored 1.00 point (95\% CI -1.58 to $0.36 ; p=0.028$ ) less on their maths GCSE exam than their healthy-weight peers, and girls who had been obese at age 11 years scored 1.66 points $(-3 \cdot 15$ to $-0 \cdot 18 ; p=0 \cdot 0021)$ less. Female individuals who had been obese at age 11 years were less likely to graduate from university than their healthyweight peers (odds ratio [OR] $0.75,95 \%$ CI 0.59 to 0.97 ; $\mathrm{p}=0.030$ ); the effect on graduation for those who had been overweight was less conclusive (OR $0.85,0.71$ to 1.01 ; $\mathrm{p}=0 \cdot 060)$. Male individuals who had been overweight scored 1.21 points $(95 \% \mathrm{CI}-2.84$ to $-0.81 ; \mathrm{p}=0.0011)$ less and those who had been obese $2 \cdot 24$ points $(95 \%$ CI -3.46 to $1.02 ; \mathrm{p}<0.0001)$ less on their GCSE maths exam than their non-obese peers, but there was no association between male childhood weight and university graduation (overweight: OR $1.07,0.95$ to $1.21 ; \mathrm{p}=0.26$; obesity: OR $0.89,0.66$ to 1.21 ; $\mathrm{p}=0 \cdot 47)$. In BCS70 and NCDS, there was a positive but insignificant relationship between overweight/obesity and cognitive performance but no significant findings for educational attainment.

Conclusion Our findings are robust to various causal methods and might help inform interventions to address this issue. Cross-cohort comparisons suggest that there might be a generational effect of overweight and obesity on educational outcomes. The youngest cohort was more susceptible to the negative consequences of childhood overweight and obesity, but the oldest cohorts were not. This needs to be explored in further research.

\section{P60 JOB STRAIN AND PERIPHERAL ARTERY DISEASE: A MULTI-COHORT STUDY}

${ }^{1} \mathrm{~K}$ Heikkila*, ${ }^{2} \mathrm{PDD}-$ Work Consortium. ${ }^{1}$ Division of Insurance Medicine, Karolinska Institutet, Stockholm, Sweden; ${ }^{2}$ Department of Public Health, University of Helsinki, Finland

\subsection{6/jech-2020-SSMabstracts.153}

Background Job strain is implicated in many cardiovascular diseases, including coronary heart disease and stroke. However, its role in peripheral artery disease (PAD), a major cause of cardiovascular morbidity worldwide, is unclear. We investigated the longitudinal association of job strain with PAD, using individual-level data from 11 prospective, register-linked cohort studies from Finland, Sweden, Denmark and the United Kingdom.

Methods Job strain (a combination of high demands and low control at work) was self-reported at baseline (1985-2008) and PAD diagnoses during the follow-up were ascertained from national hospital registers. Individuals with a pre-baseline hospital record of $\mathrm{PAD}$ were excluded from the analyses. Data on job strain, PAD and covariates were harmonised across the studies. We used Cox regression to examine the associations of job strain with PAD in each study in turn, adjusting the association estimates for age, sex, socioeconomic position, tobacco smoking, alcohol intake, body mass index and leisure time physical activity. The study-specific estimates were combined using random effects metaanalyses. Heterogeneity was quantified using $\tau$-squared and Isquared and potential sources for heterogeneity were examined by stratification and meta-regression analyses.

Results Our analyses were based on data from 139,132 men and women, aged 17-70 years. Of these participants, $32,489(23.4 \%)$ reported job strain at baseline. During 